\title{
Spontaneous charge transfer at organic-organic homointerfaces to establish thermodynamic equilibrium
}

\author{
S. Duhm, H. Glowatzki, J. P. Rabe, and N. Koch ${ }^{\mathrm{a})}$ \\ Institut für Physik, Humboldt-Universität zu Berlin, Newtonstr. 15, D-12489 Berlin, Germany \\ R. L. Johnson \\ Institut für Experimentalphysik, Universität Hamburg, D-22761 Hamburg, Germany
}

(Received 20 October 2006; accepted 15 February 2007; published online 22 March 2007)

\begin{abstract}
The energy level alignment of $\alpha, \omega$-dihexylsexithienyl (DH6T) mono- and multilayers on tetrafluorotetracyanoquinodimethane (F4-TCNQ) precovered $\mathrm{Ag}(111)$ and polycrystalline $\mathrm{Au}$ substrates was investigated with ultraviolet photoelectron spectroscopy. For certain F4-TCNQ precoverages molecular level pinning at DH6T monolayer-multilayer homointerfaces was observed. The pinning behavior shows that thermodynamic equilibrium can be established across hexyl chains via charge transfer, indicating the limited use of these short alkyl chains for insulation in the field of molecular electronics. (C) 2007 American Institute of Physics. [DOI: 10.1063/1.2715042]
\end{abstract}

The charge carrier injection barrier height across organic/metal interfaces plays a crucial role in virtually all devices in the field of "organic electronics." "-3 Energy levels at organic/metal interfaces can be tuned by precovering the metal substrate with strong electron accepting molecules such as tetrafluorotetracyanoquinodimethane (F4-TCNQ) [chemical structure shown in Fig. 1(a)]. Such molecules can undergo a charge-transfer-like interaction on the metal substrate, ${ }^{4,5}$ which increases the substrate work function. Energy levels of subsequently deposited molecules are aligned to this new effective work function. $\alpha, \omega$-dihexylsexithienyl (DH6T) [Fig. 1(a)] on $\mathrm{Ag}(111)$ and polycrystalline Au undergoes a structural transition from a flat lying monolayer (L1) to inclined multilayers (L2). ${ }^{6}$ Most likely changes in the polarization energy, as suggested to explain similar changes in orientation for pentacene, ${ }^{7,8}$ lead to a decrease of the ionization energy of the molecules and consequently to a rigid shift of molecular levels towards the Fermi level $\left(E_{F}\right)$ [i.e., lowering of the hole injection barrier (HIB)]. In our work we investigated the energy level alignment of DH6T deposited on F4-TCNQ precovered $\mathrm{Ag}(111)$ and polycrystalline $\mathrm{Au}$ with ultraviolet photoelectron spectroscopy (UPS). A transition from vacuum level alignment to molecular level pinning — reminiscent of Fermi-level pinning ${ }^{9}$ - at the homointerface between DH6T monolayer and multilayers was observed, which depended on the amount of predeposited F4-TCNQ.

UPS experiments were carried out at the FLIPPER II end station at HASYLAB (Hamburg, Germany). ${ }^{10}$ The interconnected sample preparation chambers (base pressure of $2 \times 10^{-9} \mathrm{mbar}$ ) and analysis chamber (base pressure of $2 \times 10^{-10}$ mbar) allowed sample transfer without breaking ultrahigh vacuum conditions. The $\mathrm{Ag}(111)$ single crystal was cleaned by repeated Ar-ion sputtering and annealing cycles (up to $550{ }^{\circ} \mathrm{C}$ ) until a clear low energy electron diffraction pattern was observed. The polycrystalline Au substrates were at least $400 \AA$ thick Au films on a Cu foil, evaporated in situ and Ar-ion sputtered before use. DH6T (H. C. Starck GmbH) and F4-TCNQ (Fluka) were evaporated using resistively

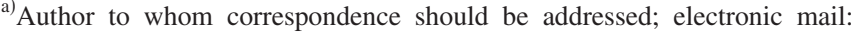
norbert.koch@physik.hu-berlin.de heated pinhole sources at evaporation rates of about $1 \AA / m i n$. The film mass thickness was monitored with a quartz crystal microbalance. Spectra were recorded with a double-pass cylindrical mirror analyzer with an energy resolution of $0.15 \mathrm{eV}$ and a photon energy of $22 \mathrm{eV}$. The second-

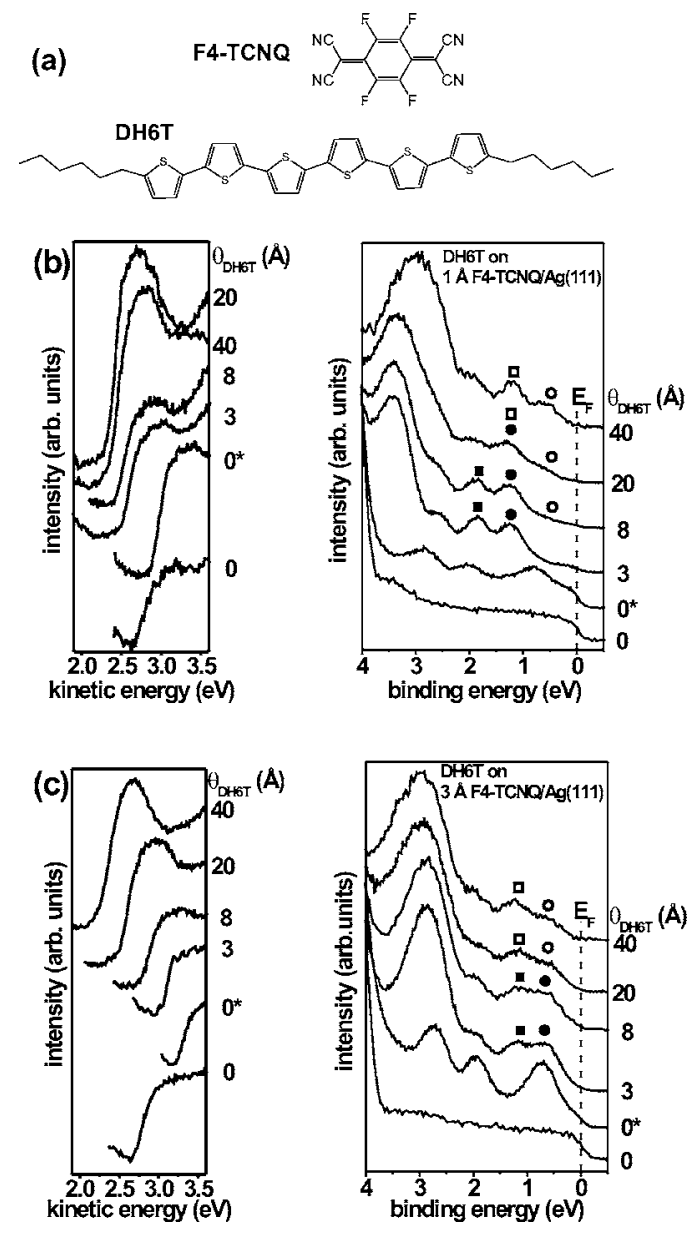

FIG. 1. (a) Chemical structure of F4-TCNQ and DH6T. (b) SECO (left side) and valence region photoemission spectrum (right side) for DH6T with increasing coverage $\left(\theta_{\mathrm{DH} 6 \mathrm{~T}}\right)$ on $1 \AA$ F4-TCNQ precovered $\mathrm{Ag}(111)$. " 0 ", is the spectrum of the F4-TCNQ precovered substrate. Closed symbols mark HOMO and HOMO-1 of L1. Open symbol mark the same features for L2. (c) Same as (b) for $3 \AA$ F4-TCNQ precoverage. 
ary electron cutoffs (SECOs) [for determination of the sample work function $(\phi)$ ] were measured with the sample biased at $-3.00 \mathrm{~V}$. To avoid sample charging during photoemission from multilayer DH6T films, spectra were recorded with an $\mathrm{Al}$ foil in the photon beam, reducing the photon flux by a factor of 7. All measurements were performed at room temperature.

Figure 1(b) shows the spectra of a DH6T thicknessdependence series on $1 \AA$ F4-TCNQ precovered $\mathrm{Ag}(111)$. F4-TCNQ chemisorbed on Ag results in three characteristic photoemission features [centered at $0.70,2.00$, and $2.80 \mathrm{eV}$ binding energies (BEs)], reminiscent of an organic-metal charge-transfer-type interaction. ${ }^{4,11}$ The shape of the UPS spectra of DH6T deposited on $1 \AA \mathrm{F} 4-\mathrm{TCNQ} / \mathrm{Ag}(111)$ was very similar to the spectra of DH6T on pristine $\operatorname{Ag}(111){ }^{6}$ However, all spectra were rigidly shifted by $0.45 \mathrm{eV}$ to lower BEs, because the DH6T molecular levels were realigned to the F4-TCNQ modified substrate surface potential. ${ }^{4}$ As reported for DH6T/Ag(111) (Ref. 6) with increasing film thickness the spectra were shifted $0.60 \mathrm{eV}$ towards lower $\mathrm{BE}$ and the intensity ratio of highest occupied molecular orbital (HOMO) and HOMO-1 was changed from approximately $1: 1$ to approximately $1: 2$. These changes in the position and line shape of DH6T spectra were already assigned before to a transition from flat lying molecules in the monolayer (L1) to inclined molecules in multilayers (L2). ${ }^{6}$ Thus, beyond $3 \AA$ DH6T coverage the formation of multilayers sets in [Fig. 1(b)]. The hole injection barrier (measured as energy difference between the HOMO onset and $E_{F}$ ) for multilayer (e.g., $40 \AA ̊)$ DH6T/Ag(111) was only $0.10 \mathrm{eV}$. Note that the SECO remained essentially at constant kinetic energy for monolayer and multilayer DH6T coverages.

Precovering $\mathrm{Ag}(111)$ with $3 \AA \mathrm{F} 4-\mathrm{TCNQ}$ led to a larger increase in sample $\phi(0.60 \mathrm{eV})$ and hence to a larger rigid shift of molecular levels of subsequently deposited DH6T [Fig. 1(c)] compared to DH6T on $1 \AA$ F4-TCNQ/ $\mathrm{Ag}(111)$. Now, even for the monolayer DH6T the HIB was only $0.10 \mathrm{eV}$. The similarity of the $3 \AA$ DH6T spectra in Figs. 1(b) and 1(c) allows us to conclude that DH6T molecules in the monolayer are lying flat on $3 \AA$ F4-TCNQ/ $\mathrm{Ag}(111)$, because (i) the formation of aggregated islands of inclined molecules at this low total coverage would not lead to the observed attenuation of substrate emission close to $E_{F}$, and (ii) the SECO would be significantly broadened due to the coexistence of surface patches with very different local work function; however, the SECO width is the same for all thicknesses. Going from L1 to L2 changed the intensity ratio of HOMO and HOMO-1 from almost 1:1 to approximately $1: 2$, which again is interpreted as evidence for inclined molecules. Noteworthy, this change was not accompanied by a further decrease of the HIB. Also the SECO showed a different behavior compared to DH6T on $1 \AA$ F4-TCNQ/ $\mathrm{Ag}(111)$. Even after monolayer completion the SECO continued to shift towards lower kinetic energies and reached the same position for the multilayers as for the case shown in Fig. 1(b).

Figure 2 summarizes these results. Part (a) indicates the growth model of DH6T on pristine $\mathrm{Ag}(111)$ (left side) and DH6T on F4-TCNQ precovered $\mathrm{Ag}(111)$ (right side). Part (b) displays the corresponding schematic of the interface electronic structure. Note that the energy level alignment of the DH6T homointerface L1/L2 was the same for DH6T/ $\operatorname{Ag}(111)$ and DH6T on $1 \AA$ F $4-\mathrm{TCNQ} / \mathrm{Ag}(111)$, however, Downloaded 14 Aug 2007 to 131.169.95.147. Redistribution subje

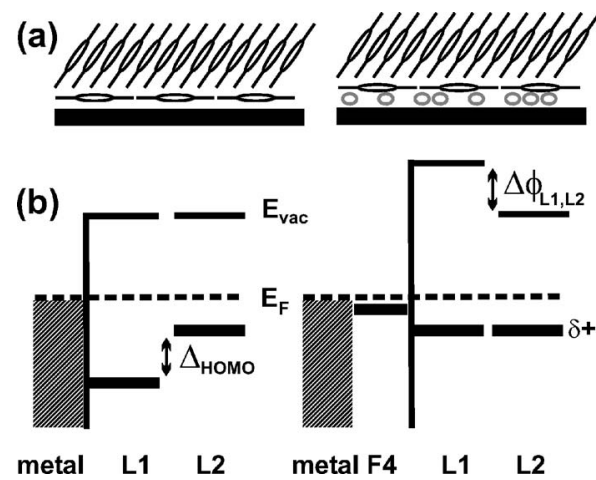

FIG. 2. (a) Growth model and (b) interface electronic structure model for DH6T/Ag(111) (left side) and DH6T/(thick)F4-TCNQ/Ag(111) (right side). L1 indicates monolayer DH6T, L2 multilayer DH6T, $E_{\text {vac }}$ vacuum level, $E_{F}$ Fermi level, $\Delta \phi_{\mathrm{L} 1, \mathrm{~L} 2}$ the work function shift, $\Delta_{\mathrm{HOMO}}$ the HOMO shift, $\delta+$ symbolizes the slightly charged L2, and F4 means F4-TCNQ.

rigidly shifted. For low (or even zero) $\theta_{\mathrm{F} 4-\mathrm{TCNQ}}$ (left side) the vacuum level $\left(E_{\text {vac }}\right)$ remained constant at the DH6T homointerface L1/L2, but the DH6T HOMO shifted towards $E_{F}$ $\left(\Delta_{\text {HOMO }}\right)$, i.e., the ionization energy of L2 was smaller than for L1. On the other hand (right side), for $\theta_{\mathrm{F} 4-\mathrm{TCNQ}}>3 \AA$ the hole injection barrier stayed constant at the L1/L2 interface and $E_{\mathrm{vac}}$ (vacuum level) exhibited a shift $\left(\Delta \phi_{\mathrm{L} 1, \mathrm{~L} 2}\right)$. This behavior is typical for molecular orbital level pinning. ${ }^{9}$ It has been suggested that the transition from vacuum level alignment to molecular orbital level pinning occurs when the HIB becomes of the same magnitude as the positive polaron relaxation energy. ${ }^{9,12,13}$ In this case, charge must be redistributed across the interface, leading to an interface dipole. In other words, the vacuum level is changed but the hole injection barrier stays constant. The measured vacuum level shift $\Delta \phi_{\mathrm{L} 1, \mathrm{~L} 2}$ is direct evidence for an interface dipole and the accompanied charge redistribution across the DH6T homointerface L1/L2. Remarkably, our results demonstrate that the insulating hexyl chains do not obstruct this charge transfer but allow for thermodynamic equilibrium to be established. But, since the arrangement of hexyl chains and conjugated backbone in the multilayer is not known, charge does not have to flow necessarily along the hexyl chains.

To extend the above results obtained on the model substrate $\operatorname{Ag}(111)$ to a more application relevant one, similar experiments were performed using polycrystalline Au as substrate. On pristine $\mathrm{Au}$, DH6T showed the same rigid shift (as on $\mathrm{Ag}$ ) of molecular levels when going from L1 to L2 [Fig. 3(a), upper part]. For $1 \AA$ F4-TCNQ precoverage, already the monolayer DH6T (L1) had a hole injection barrier of only $0.10 \mathrm{eV}$. Note that this corresponds to the situation observed for $3 \AA$ F4-TCNQ precoverage on $\mathrm{Ag}(111)$ [Fig. $1(c)$ ], indicating that the F4-TCNQ coverage dependence differs for the two rather dissimilar metal surfaces. Multilayer formation did not lead to a further reduction of the HIB [Fig. 3(a), bottom part]. Changes in the hole injection barrier and the vacuum level for lower F4-TCNQ precoverages are summarized in Fig. 3(b). The upper part displays the HIB of L1 and L2 of DH6T as function of F4-TCNQ precoverage $\left(\theta_{\mathrm{F} 4-\mathrm{TCNQ}}\right)$. The bottom part displays the corresponding vacuum level shift $\left(\Delta_{\text {vac }}\right)$ with respect to pristine $\mathrm{Au}$. Multilayer DH6T on pristine Au had the same $\Delta_{\text {vac }}$ as monolayer DH6T, but a decreased HIB, equivalent to DH6T/ $\mathrm{Ag}(111)$ [Fig. 2(b)]. For F4-TCNQ precovered Au with a monolayer of DH6T, the HIB depended almost linearly on to AIP license or copyright, see http://apl.aip.org/apl/copyright.jsp 

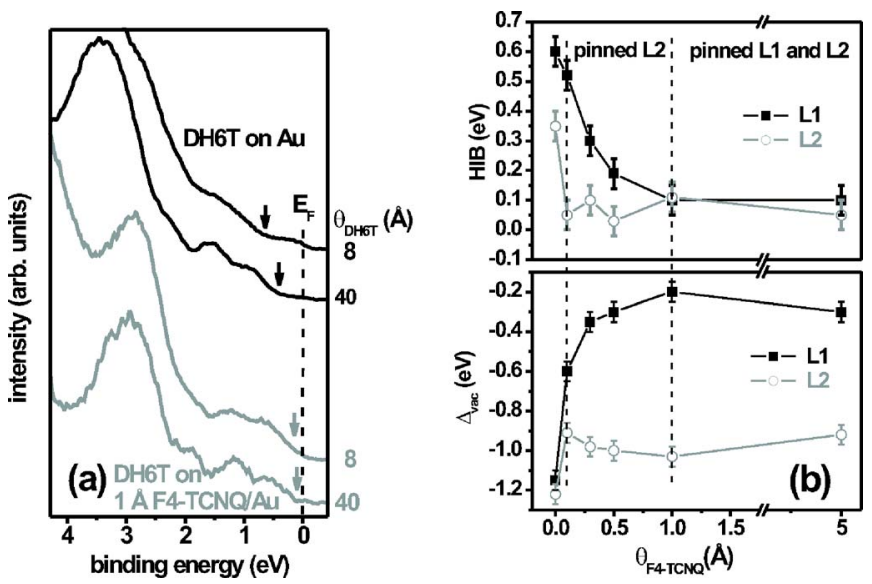

FIG. 3. (a) UPS spectra of DH6T/Au (upper spectra) and DH6T on $1 \AA$ F4-TCNQ/Au (bottom spectra), $\theta_{\mathrm{DH} 6 \mathrm{~T}}$ is the DH6T layer thickness, and the arrows indicate the HOMO onset. (b) Hole injection barrier and vacuum level shift $\left(\Delta_{\text {vac }}\right)$ (referenced to the vacuum level of pristine $\mathrm{Au}$ ) of a monolayer (filled squares) and multilayer (open circles) DH6T on F4-TCNQ/Au films as function of F4-TCNQ precoverage thickness $\left(\theta_{\mathrm{F} 4-\mathrm{TCNQ}}\right)$. The dashed lines symbolize the boundary between the different pinning regimes.

$\theta_{\text {F4-TCNQ }}$ until $0.5 \AA$ was reached, leveled off between 0.5 and $1 \AA$, and remained constant between 1 and $5 \AA$. Apparently, also the vacuum level shift was reduced until $1 \AA$ F4TCNQ precoverage and stayed essentially constant further on. The constant values for the thick DH6T film point to molecular level pinning. In this case the alignment of molecular orbitals is controlled by the molecular polaronic level and not by the effective substrate work function. One can distinguish three different energy level alignment mechanism regimes of DH6T as function of F4-TCNQ precoverage. (i) On pristine $\mathrm{Au}$ and on $0.10 \AA \mathrm{F} 4-\mathrm{TCNQ} / \mathrm{Au}$ the molecular levels of L1 and L2 were vacuum level controlled. (ii) From 0.10 to $1 \AA \mathrm{F} 4-\mathrm{TCNQ} / \mathrm{Au} \mathrm{L} 1$ was still vacuum level controlled, but L2 was molecular level pinned. (iii) For $\theta_{\mathrm{F} 4-\mathrm{TCNQ}}$ larger than $1 \AA$ both L1 and L2 were pinned.

One can estimate the average charge transfer per molecule using the Helmholtz equation: $\Delta \phi_{\mathrm{L} 1, \mathrm{~L} 2}=q n_{D} \mu / \varepsilon \varepsilon_{0}$ and the DH6T molecular area density $n_{M} \cdot\left(\Delta \phi_{\mathrm{L} 1, \mathrm{~L} 2}\right.$, measured change in the work function at the monolayer/multilayer DH6T interface; $q$, elementary charge; $n_{D}$, dipole surface density; $\mu$, dipole moment perpendicular to the surface; $\varepsilon$, dielectric constant; and $\varepsilon_{0}$, vacuum permittivity.) $\Delta \phi_{\mathrm{L} 1, \mathrm{~L} 2}$ was measured to be $0.85 \mathrm{eV}$ on both metal substrates. The layer thickness of L2 is known to be $26 \AA$ for DH6T/ $\mathrm{Ag}(111)$ (Ref. 6) and assumed to be similar for DH6T/Au. For our calculations, we used half of this value to estimate the distance between the charges giving rise to $\Delta \phi_{\mathrm{L} 1, \mathrm{~L} 2}$. We chose $\varepsilon=3$, which is a commonly accepted value for conjugated organic materials. ${ }^{14}$ The crystal structure of DH6T is unknown. Thus, $n_{M}$ was estimated from the crystal structure of 6T (Ref. 15), with $n_{M}=3.92 \times 10^{14} \mathrm{~cm}^{-2}$. The ratio of $n_{D}$ and $n_{M}$ gives for the average charge per DH6T molecule in the multilayer $0.03 q$. This relatively small value can explain why no new density of states (i.e., corresponding to polaronic levels) was observed close to $E_{F}$ by photoemission.

Photoemission results of DH6T/F4-TCNQ/metal heterostructures have shown that for high work function electrodes (in this work metals covered with strong electron acceptors) the positive polaron relaxation energy of multilayer DH6T film is in the range of the DH6T hole injection barrier. In this case, the energy level alignment changes from being vacuum level controlled to molecular orbital level pinning. The measured shift in the vacuum level between monolayer and multilayer DH6T is direct evidence for interface dipoles and for charge transfer between molecular layers. The observed pinning behavior suggests that hexyl chains are not appropriate insulating layers for the use in molecular electronics, and longer chains may be needed.

The authors thank H. C. Starck GmbH for providing DH6T. One of the authors (N.K.) acknowledges financial support by the Emmy Noether Program (DFG).

${ }^{1}$ A. Kahn, N. Koch, and W. Gao, J. Polym. Sci., Part B: Polym. Phys. 41, 2529 (2003).

${ }^{2}$ H. Ishii, K. Sugiyama, E. Ito, and K. Seki, Adv. Mater. (Weinheim, Ger.) 11, 605 (1999).

${ }^{3}$ W. R. Silveira and J. A. Marohn, Phys. Rev. Lett. 93, 116104 (2004).

${ }^{4}$ N. Koch, S. Duhm, A. Vollmer, R. L. Johnson, and J. P. Rabe, Phys. Rev. Lett. 95, 237601 (2005).

${ }^{5}$ S. Duhm, H. Glowatzki, V. Cimpeanu, J. Klankermayer, J. P. Rabe, R. L. Johnson, and N. Koch, J. Phys. Chem. B 110, 21069 (2006).

${ }^{6}$ S. Duhm, H. Glowatzki, J. P. Rabe, N. Koch, and R. L. Johnson, Appl. Phys. Lett. 88, 203109 (2006).

${ }^{7}$ H. Fukagawa, H. Yamane, T. Kataoka, S. Kera, M. Nakamura, K. Kudo, and N. Ueno, Phys. Rev. B 73, 245310 (2006).

${ }^{8}$ N. Koch, I. Salzmann, R. L. Johnson, J. Pflaum, R. Friedlein, and J. P. Rabe, Org. Electron. 7, 537 (2006).

${ }^{9}$ C. Tengstedt, W. Osikowicz, W. R. Salaneck, I. D. Parker, C.-H. Hsu, and M. Fahlman, Appl. Phys. Lett. 88, 053502 (2006).

${ }^{10}$ R. L. Johnson and J. Reichardt, Nucl. Instrum. Methods Phys. Res. 208, 791 (1983).

${ }^{11}$ N. Koch, A. Rajagopal, J. Ghijsen, R. L. Johnson, G. Leising, and J.-J. Pireaux, J. Phys. Chem. B 104, 1434 (2000).

${ }^{12}$ P. S. Davids, A. Saxena, and D. L. Smith, Phys. Rev. B 53, 4823 (1996).

${ }^{13}$ N. Koch and A. Vollmer, Appl. Phys. Lett. 89, 162107 (2006).

${ }^{14}$ I. G. Hill, A. Kahn, Z. G. Soos, and R. A. Pascal, Jr., Chem. Phys. Lett. 327, 181 (2000).

${ }^{15}$ T. Siegerist, R. M. Fleming, R. C. Haddon, R. A. Laudise, A. J. Lovinger, H. E. Katz, P. Bridenbaugh, and D. D. Davis, J. Mater. Res. 10, 2170 (1995). 